\title{
XIX. On the thermodynamical theory of radiation
}

\section{J.h. Jeans}

To cite this article: J.h. Jeans (1907) XIX. On the thermodynamical theory of radiation, Philosophical Magazine Series 6, 13:74, 285-286, DOI: 10.1080/14786440709463602

To link to this article: http://dx.doi.org/10.1080/14786440709463602

册 Published online: 16 Apr 2009.

Submit your article to this journal $₫$

View related articles $\sqsubset$ 
propagated with the velocity of light. The ratio $d t_{v} / d t$ is thus found to be introduced as a denominator into the velocity of the oscillating mass, since Doppler's factor modifies the duration of the oscillation propagated. Now the momentum being proportional to the velocity, it is momentum in the electromagnetic field that fulfils the requirements outlined above, and this involves the assumption that field-intensity is of the nature of momentum.

In a memoir published by Bjerknes*, on the analogy between the steady electromagnetic field and the bydrodynamic field, the author shows that field-intensity in the electromagnetic field is analogous to momentum in the hydrodynamic one. There is between his conclusions, to which I would draw attention, and the necessity of introducing Doppler's factor, a concordance which is worthy of notice.

XIX. On the Thermodynamical Theory of Radiation. To the Editors of the Philosophical Magazine.

\section{Gentlemen, -}

Mr. Tuckerman's criticisms $\dagger$ appear to be based on a misunderstanding of my position. He brings as an objection to my argument, that the temperature $\mathrm{T}$ is " a quantity which is and can be defined only in terms of the properties of matter."

My argument was directed against the thermodynamical proof of the laws of radiation. It is therefore sufficient for my reductio ad absurdum that according to the thermodynamical theory underlying this proof it is possible to attribute temperature to the æther. (In the thermodynamical proof in question we are concerned with an imaginary heatengine, using xther as working substance, and working between temperatures $\mathrm{T}_{1}$ and $\mathrm{T}_{2}$.)

Apart from this, however, I wish to take this opportunity of remarking that $i t$ is quite easy to eliminate the idea of temperature completely from my original paper $\ddagger$. Let us agree to measure the warmth of the radiator by a quantity $\mathrm{E}$, which is to be equal to the average energy of a principal degree of freedom in the radiator, and therefore in the ordinary notation, to $\frac{1}{2} \mathrm{RT}$.

The radiation-function $\phi$ must be a function of $\lambda, \mathrm{E}, \mathrm{V}, e$, $m$, and $\mathrm{K}$. There are now six independent quantities and four independent physical units, namely those of length, mass, time, and inductive capacity. As before, we can combine the six quantities in two independent ways so as to

* Archives des Sciences phys. et nat. 1905, t. xx. p. 325.

† Phil. Mag. Nov. 1906, p. 498.

$\ddagger$ Proc. Roy. Soc. A. Ixxvi. p. 545.

Plit. Mag. S. 6. Vol. 13. No. 74. Feb. 1907. 
obtain a pure number, say

$$
c_{1}=\mathrm{E} m^{-1} \mathrm{~V}^{-2}, \quad c_{2}=\lambda \mathrm{EK} e^{-2} ;
$$

and, in order that $\phi$ may be of the dimensions of energy per unit volume per unit wave-length, we find that $\phi$ must be of the form

$$
\phi=\lambda^{-4} \mathrm{E} f\left(c_{1}, c_{2}\right)
$$

whence, on passing to the limit as before, we obtain

$$
\phi=\lambda^{-4} \mathbf{E} f(\lambda E) \text {. }
$$

Stefan's formula follows on integration with respect to $\lambda$ in the form $\mathrm{SE}^{4}$, the value $\mathrm{S}$ being of course identical with the former $16 \mathrm{R}^{-4} \sigma$.

Hence it appears that the argument can be modified without trouble, so that the conception of temperature disappears altogether, being replaced by the conception of energy. The objection brought against my original argument, namely, that "temperature is and can be defined only in terms of the properties of matter, and in fact in terms of properties which are common to all matter," must, I think, be withdrawn when "energy" is substituted for "temperature."

J. H. JEANS.

Princeton, N.J.

XX. Notices respecting New Books.

The Electron Theory. A Popular Introduction to the ncw Theory of Electricity and Maynetism. By E. E. Fournier D'ALBE, B.Sc., A.R.C.Sc. With a Preface by G. Johnstone Stonex, M.A., Sc.D., F.R.S. London: Longmans, Green, \& Co. 1906. Pp. xxiv +312.

THE great interest which is being evinced by all educated people in the new views regarding the nature of matter that have been forced upon the scientific world by the brilliant researches of the last decade should secure a wide circulation for this book. In simple and easy language, free from unnecessary technicalities, the author presents to his readers an account of the electron theory, and shows how the theory may be made to interpret in a satisfactory manner all the old familiar facts, as well as discoveries of more recent origin. A brief sketch of the development of the electron theory is first given, and is followed by an account of the elementary facts of electricity and magnetism, couched in terms suitable to the new conceptions introduced by the electron theory. This part of the book is, in a sense, an elementary text-book written on entirely up-to-date lines. $\boldsymbol{A}$ commendable feature is the frequent use of arithmetical calculation for the purpose of arriving at results which give the reader a correct notion of the magnitude of the various effects discussed. The later chapters are mainly devoted to recent advances, and include an account of the electromagnetic theory of light, of magneto-optic phenomena, of radio-activity, and a discussion of the constitution of the 